\title{
A model of sound scattering by atmospheric turbulence for use in noise mapping calculations
}

\author{
Jens Forssén ${ }^{1}$, Maarten Hornikx ${ }^{1,2}$, Dick Botteldooren ${ }^{3}$, Weigang Wei $^{3}$, Timothy Van \\ Renterghem $^{3}$ and Mikael Ögren ${ }^{4}$ \\ ${ }^{1)}$ Department of Civil and Environmental Engineering, Division of Applied Acoustics, \\ Chalmers University of Technology, SE-41296 Göteborg, Sweden. \\ ${ }^{2)}$ Building Physics and Services, Eindhoven University of Technology, Eindhoven, the Netherlands \\ ${ }^{3)}$ Department of Information Technology, Ghent University, Ghent, Belgium \\ ${ }^{4)}$ Department of Environmental and Traffic Analysis, VTI, Gothenburg, Sweden
}

\begin{abstract}
Summary
Sound scattering due to atmospheric turbulence limits the noise reduction in shielded areas. An engineering model is presented, aimed to predict the scattered level for general noise mapping purposes including sound propagation between urban canyons. Energy based single scattering for homogeneous and isotropic turbulence following the Kolmogorov model is assumed as a starting point and a saturation based on the von Kármán model is used as a first-order multiple scattering approximation. For a single shielding obstacle the scattering model is used to calculate a large dataset as function of the effective height of the shielding obstacle and its distances to source and receiver. A parameterisation of the dataset is used when calculating the influence of single or double canyons, including standardised air attenuation rates as well as façade absorption and Fresnel weighting of the multiple façade reflections. Assuming a single point source, an averaging over three receiver positions and that each ground reflection causes energy doubling, the final engineering model is formulated as a scattered level for a case without canyon and a correction term for the effect of a single or a double canyon case, assuming a flat rooftop of the shielding building. Input parameters are, in addition to geometry and sound frequency, the strengths of velocity and temperature turbulence.
\end{abstract}

PACS no. 43.20.Hq, 43.28.Js

\section{Introduction}

When acoustic shadow regions appear, enhancing areas with sound weaker than the free field level, the sound scattering by turbulence grows in importance. The shadow regions of interest here are those caused by shielding objects such as buildings and other noise barriers. Acoustic shadows caused by upward refraction are similarly affected but not focus of the current study. The turbulence of the atmospheric surface layer has previously been shown to increase the noise level behind barriers, mainly at higher sound frequencies (e.g. [1]). In first estimates, the turbulent flow actually caused by a noise barrier itself, has been shown to lead to less significant scattering [2]. Previous studies have shown that models using energy based single scattering approximations are well applicable to the problem (e.g. $[3,4]$ ). Even though a higher precision is expected by using wave-based models, as the parabolic equation method (e.g. [5, 6]), the finite-difference time-domain method (e.g. [7]) or the equivalent sources method [8], the single scattering approximation is considered to be accurate enough to serve as basis for an engineering model; in addition, having large benefits in computational cost. The scattering model developed in [4], based on [3] and on theory known from literature (e.g. [9, 10, 11]), has been used, in simplified forms, in engineering models for noise mapping purpose $[12,13]$. In the present paper the aim is to present an engineering model that is more generally applicable, i.e. for built up areas with street canyons and inner yards, in addition to a single screen on ground. Below, we describe the underlying scattering cross section model, the development of a numerically efficient model for non-canyon situations, a parameter study for canyon situations and the suggested engineering model for general urban situations, followed by conclusions. 


\section{Model development}

\subsection{Underlying scattering cross section model}

Using the scattering cross section by Tatarskii [9], Daigle [3] created a model for the total scattering into the shadow region created by a noise barrier, as briefly described here for convenience. The scattered intensity, or here rather the mean square acoustic pressure, $\tilde{p}^{2}$, can be written as an integral over a volume $V$, as

$$
\tilde{p}^{2}=\int_{V} \tilde{p}_{0}^{2} \frac{\sigma(\theta)}{r^{2}} \mathrm{~d} V
$$

where $p_{0}$ is the incoming, undisturbed pressure from the source, $\sigma(\theta)$ the scattering cross section as a function of the scattering angle $\theta$, and $r$ the distance from the point in volume $V$ to the receiver, where $V$ is defined as all points above the lines of sight from both the source and the receiver to the barrier top (see [3] for further details). Ostashev describes the derivation of the scattering cross section as well as different turbulence models [11]. For the work made here, a homogeneous and isotropic von Kármán turbulence model has been used. Within the inertial range of the turbulence, the scattering cross section is identical to the one for the more simplified Kolmogorov model, which can be written

$\sigma(\theta)=0.03 k^{1 / 3} \frac{\cos ^{2} \theta}{\sin (\theta / 2)^{11 / 3}}\left(\frac{C_{v}^{2}}{c_{0}^{2}} \cos ^{2} \frac{\theta}{2}+0.14 \frac{C_{T}^{2}}{T_{0}^{2}}\right)$,

where $k$ is the acoustic wave-number $\left(k=2 \pi f / c_{0}\right.$, with $f$ the sound frequency and $c_{0}$ the mean sound speed), $C_{v}$ and $C_{T}$ the structure parameters of velocity and temperature fluctuations, respectively, describing their partial turbulence strengths, and $T_{0}$ the mean temperature in Kelvin.

\subsection{Development of a turbulence scattering model for non-canyon situations}

Inherent in the above described modelling is the assumption of a single scattering approximation. In an improved model the incoming pressure, $\tilde{p}_{0}$, in Eq. (1), would be altered due to multiple scattering as well as due to the barrier diffraction. A first order correction for multiple scattering could be to remove the intensity from the incoming field that is estimated to already have been lost due to scattering by volume elements closer to the source. Here, however, a slightly different approach has been taken, where the scattering is limited by a saturation determined by an assumed smallest value of turbulence strength, as further described below. In addition, for use in a noise mapping model, the scattering should be limited so that scattered plus diffracted intensity does not exceed that of the open field, i.e. without barrier.

To reduce the numerical cost for evaluating the integral of Eq. (1), the integration is made analytically for constant $\theta$-values, i.e. in the azimuthal direction to the source-receiver line, as described previously [14]. Furthermore, since the integrand is a relatively slowvarying function of space, a fine discretization is not needed. Here a grid spacing of $1 \mathrm{~m}$ has been used, and the height and length of the integration domain is limited to about the size of the source-receiver distance.

It is evident from Eqs. (1-2) that, if the two terms corresponding to velocity and temperature fluctuations are kept separate, the integrals can be calculated for a given geometry, and the dependence on the factors $k^{1 / 3}, C_{v}^{2}$ and $C_{T}^{2}$ can be inferred later.

The effects of varying the sound frequency and the strengths of velocity and temperature turbulence as well as modelling the air attenuation and the scattering saturation are studied at a later stage. First, the total scattered level is estimated, relative to free field, for a set of geometries and for unit turbulence strengths $\left(C_{v}^{2}=1 \mathrm{~m}^{4 / 3} / \mathrm{s}^{2}\right.$ respectively $C_{T}^{2}=1 \mathrm{~K}^{2} / \mathrm{m}^{2 / 3}$ ). In the set of geometries, the screen height, $h$, is varied in $M=20$ logarithmic steps from 4 to $80 \mathrm{~m}$. The distances to the screen, from the source, $d_{S}$, as well as from the receiver, $d_{R}$, are each varied in $N=25$ logarithmic steps from 10 to $500 \mathrm{~m}$. Thereby a dataset of $M \times N \times N=12500$ cases is created (the actual number of calculations is 6500 since only the upper triangle of each $N \times N$ matrix needs to be calculated, due to symmetry).

For each source-screen distance, a planar fit is made to the scattered level as function of the $M \times N$ points of varying screen height and screen-receiver distance (in log coordinates). Since a plane can be described by a $3 \times 1$ vector of coefficients, these vectors are computed and stored for each of the $N$ planes of sourcescreen distances. Their values are appended in Tables III and IV [should be referred to as Tables A1 and A2], for velocity and temperature turbulence, respectively, where the geometric variables have been normalized by $d_{S}$, which turns out to be preferable for later use. When the result for a new geometry is to be calculated, an interpolation between the set of vectors can be made for the wanted source-screen distance, and the found $3 \times 1$ vector of plane coefficients can be used to estimate the scattered level for the screen height and screen-receiver distance of interest. If the source and the receiver are not on the same height, the input geometry to the model is first rotated. (The geometry is shown in Figure 1.) An example estimate of scattered levels were calculated assuming a source-screen distance of $d_{S}=40 \mathrm{~m}$. The interpolation then uses values at $d_{S}=36.8$ and $43.4 \mathrm{~m}$, which are the two nearest $d_{S}$ values used in the precalculation of the data set. The results are compared with those of a di- 
rect calculation for $d_{S}=40 \mathrm{~m}$, as shown in Figures 2 and 3. The maximum errors for these results are less than $3 \mathrm{~dB}$ for screen heights varying between 5 and $40 \mathrm{~m}$, and screen-receiver distances varying between 10 and $100 \mathrm{~m}$, for both velocity and temperature turbulence. The mean error is within $\pm 0.2 \mathrm{~dB}$ and the standard deviation of the error (i.e. the standard error) is about $1 \mathrm{~dB}$. Hence, the model based on this precalculated dataset can be used for calculating the amount of turbulence scatting in non-canyon cases, i.e. with a single obstacle (a building or other noise barrier) and no further reflecting façades.

\subsection{Parameter study for urban canyon situa- tions}

For the canyon situations, flat roofs have been assumed and the default cases have equal roof height. Looking at Figure 4, where the geometric parameters are explained, the default double canyon cases have $H_{S}=H_{R}=H_{I}$, whereas for single canyon cases either $H_{S}$ or $H_{R}$ is zero, and for cases without canyon, both $H_{S}$ and $H_{R}$ are zero. In the parameter study, the sound frequency and the geometric parameters were varied including three horizontally spaced receiver positions. The number of parameters, their range of values and other input data are shown in Table II.

Entirely, the set of calculations consisted of 225792 separate cases, including the 8 frequencies. To calculate the scattered level, relative to free field, for each case, the scattering is added energy wise for the different reflection orders. Reflection order zero means that the sound has not been reflected in any façade; reflection order one means one façade reflection, in either source or receiver canyon; etc. The reflections are reduced by assuming an energy absorption coefficient of the façades of $\alpha=0.2$, independent of frequency. An additional cause for energy reduction at reflection is modelled by a Fresnel number criterion, which reduces the reflections that are sufficiently close to the edge between façade and roof. For this model, the Nord2000 methodology for vertical surfaces has been used [12, Section 5.20], except an adaptation to an energy scattering based model (by using $10 \log _{10}(S)$ instead of $20 \log _{10}(S)$, where $S$ is the effective surface within the Fresnel-zone). The effect of ground is modelled as a doubling of energy both at the source side and at the receiver side. The used receiver height is $y_{R}=1.5 \mathrm{~m}$ and can be seen as an approximation also for the commonly used receiver height of $4 \mathrm{~m}$. In the calculations, reflections up to order $m=15$ were used, which, for these settings, was shown by numerical tests to give converging results.

Since the single scattering approximation leads to overprediction at longer distances, a saturation of the scattering is modelled. This is done by multiplying the scattered energy by $\exp \left(-2 x k^{2} J_{\text {vonK }}\right)$, where $x$ is the horizontal range of propagation and $J_{\mathrm{vonK}}=10^{-8} \mathrm{~m}$.
Here, $k^{2} J_{\text {vonK }}$ is the total extinction coefficient according to the von Kármán model [11], and the value of $J_{\text {vonK }}$ has been estimated from assuming a rather small outer length scale of $L_{0}=10 \mathrm{~m}$ and small values of the structure parameters, such that $C_{v}^{2} / c_{0}^{2}$ and $C_{T}^{2} / T_{0}^{2}$ approximately equals $10^{-8} \mathrm{~m}^{-2 / 3}$ in the expression

$$
J_{\mathrm{vonK}}=\frac{3}{10} \pi^{2} A K_{0}^{-5 / 3}\left(4 \frac{C_{v}^{2}}{c_{0}^{2}}+\frac{C_{T}^{2}}{T_{0}^{2}}\right)
$$

where $K_{0}=2 \pi / L_{0}$.

Furthermore, the effect of air attenuation is taken into account, with a level reduction in proportion to the horizontal range, $x$, using standardized attenuation rates as function of frequency ${ }^{1}$.

When calculating the contribution of each reflection, the scattered level for the corresponding path in the non-canyon situation is first found by interpolation between the pre-calculated set of vectors (from Tables III and IV). Then the effects of façade absorption, Fresnel weighting, air attenuation and scattering saturation are included before the contributions from all reflections are added.

\subsection{Engineering turbulence scattering model for general urban situations}

For the engineering models, the results from the parameter study are first energy averaged over the three horizontally separated receiver positions. One quarter of the calculated cases are for both $H_{S}$ and $H_{R}$ being zero, i.e. situations without any canyon. It turns out that these 18816 cases are well approximated by a linear fit of geometric variables $\log _{10} \frac{h}{d_{0}}$ and $\log _{10} \frac{h^{2}}{d_{S} d_{R}}$, in addition to $\frac{10}{3} \log _{10} \frac{f}{f_{0}}$, where $d_{0}=10 \mathrm{~m}$ and $f_{0}=1000 \mathrm{~Hz}$ have been used and where the geometrical distances, as depicted in Figure 1, now are interpreted as the effective distances from the source to the mid receiver position over a thin screen. The resulting model for the scattered level, relative to free field, in situations without canyon, $L_{p}$, scat, no canyon, is written as follows.

$$
\begin{aligned}
& L_{p, \text { scat }, \text { no canyon }}=b_{1}+b_{2} \log _{10} \frac{h}{d_{0}} \\
& +b_{3} \log _{10}\left(\frac{h^{2}}{d_{S} d_{R}}+\epsilon\right)+\frac{10}{3} \log _{10} \frac{f}{f_{0}}
\end{aligned}
$$

where the values of $b_{i}(i=1,2,3)$ are given in Table I and where $\epsilon$ is inserted in order to reduce the scattered level when outside of the domain used in

\footnotetext{
${ }^{1}$ Applying values from ISO 9613, part 1, for standard atmospheric conditions with a relative humidity of $70 \%$, a temperature of $20^{\circ} \mathrm{C}$ and a static pressure of $101325 \mathrm{~Pa}$.
} 
the parameter study, with a value of 0.0012 , given by numerical tests. Comparing the engineering model with the detailed results of the parameter study, the standard error is about $2 \mathrm{~dB}$ for both velocity and temperature turbulence.

The derived model of the scattered level in the canyon case, $L_{p}$, scat, canyon, is given as a correction term to the level for the non-canyon case:

$$
L_{p, \text { scat, canyon }}=L_{p, \text { scat, no canyon }}+\Delta L_{\gamma} .
$$

The correction term $\Delta L_{\gamma}$ is estimated as follows (with $H_{0}=10 \mathrm{~m}$ ).

$$
\begin{aligned}
& \Delta L_{\gamma}=\gamma_{1}+\gamma_{2} \log _{10} \frac{H_{I}}{H_{0}}, \\
& \gamma_{1}= \begin{cases}7, & \text { if single canyon } \\
14, & \text { if double canyon }\end{cases} \\
& \gamma_{2}=\left\{\begin{array}{l}
2 H_{I} / W_{S}, \text { if single canyon, on source side } \\
2 H_{I} / W_{R}, \text { if single canyon, on receiver side } \\
2 H_{I}\left(1 / W_{S}+1 / W_{R}\right), \text { if double canyon }
\end{array}\right.
\end{aligned}
$$

For the above model with canyons, the standard deviation of the error is about $6 \mathrm{~dB}$, for both the velocity and temperature turbulence scattering. Even though further accuracy improvements of the model are possible, the balance between simplicity and accuracy is deemed appropriate for the purpose of engineering noise map calculation models.

For an intermediate height of $H_{S}$ or $H_{R}$, i.e. between 0 and $H_{I}$, it is suggested that a linear interpolation of the level is used. Calculated results (not presented here) have shown that the scattered level is a monotonically increasing function with the height of $H_{S}$ or $H_{R}$. The rate of increase is higher closer to $H_{I}$, whereby the linear interpolation corresponds to a conservative estimate in the sense of rather overestimating than underestimating the scattered level. Furthermore, as $H_{S}$ or $H_{R}$ approaches $H_{I}$, the level converges toward a maximum, whereby results for values of $H_{S}$ or $H_{R}$ larger than $H_{I}$ can be taken as those at $H_{I}$.

Suggested starting values of the structure parameters for fairly strong turbulence are $C_{v}^{2}=1.2 \mathrm{~m}^{4 / 3} / \mathrm{s}^{2}$ and $C_{T}^{2}=0.4 \mathrm{~K}^{2} / \mathrm{m}^{2 / 3}$. It could be noted that, in relation to typical values in literature, the values suggested here are relatively large; however motivated by previous results [4], where measured values of the structure parameters in a setting with a thick noise barrier were used as input to scattering predictions of the sound field, which were compared with measured acoustic data. The suggested values are also in the same order as those found in a recent study [15]. Furthermore, in [16] the model described here, together with the suggested turbulence strength, have been used for three shielded urban cases, resulting in a largely improved agreement with measured data compared with assuming no scattering by turbulence.

Since the model presented here assumes a point source in a domain that varies only in two dimensions, it is suggested that a so-called $2.5 \mathrm{D}$ approach is used for sources further down the road, and the width of the intermediate building is taken as the length of the source-receiver line occupied by the building. It could also be stressed that, for use in noise mapping models, the scattering should be limited so that the total level including diffraction does not exceed that predicted for open field.

\section{Conclusion}

A previously established turbulence scattering cross section model for a single noise screen has been used to develop an engineering model for a general urban situation with the possibility to account for a street canyon and an inner yard, assuming homogeneous and isotropic turbulence. As an intermediate step, a numerically efficient model was developed, which was also made to account for multiple facade reflections, and then used for a parameter study. Using the results of the parameter study, the engineering model was developed with the aim to balance computational cost and accuracy. Studying the error for the case without canyons, the engineering model showed an overall standard deviation of about $2 \mathrm{~dB}$ in relation to the intermediate model, which in turn showed an error of about $1 \mathrm{~dB}$ in relation to the starting model. Hence, by assuming additivity of the variances, the total error can be estimated to have a standard deviation of less than $3 \mathrm{~dB}$. With canyons the standard deviation of the error increases further, up to about $6 \mathrm{~dB}$.

\section{Acknowledgements}

Part of this work was financially supported by the Life + program of the European Community (project QSIDE, LIFE09 ENV/NL/000423).

\section{References}

[1] Sound shielding in the presence of turbulence (Special issue). Applied Acoustics, Vol. 65, Issue 6, 2004, pp. 559-642.

[2] Heimann, D. and Blumrich, R. Time-domain simulations of sound propagation through screen-induced turbulence. Applied Acoustics, Vol. 65, 2004, pp. 561582 .

[3] Daigle GA. Diffraction of sound by a noise barrier in the presence of atmospheric turbulence. J. Acoust. Soc. Am. 1982, Vol. 71, pp. 847-54. 
[4] Forssén J. and Ögren, M. Barrier noise-reduction in the presence of atmospheric turbulence: Measurements and numerical modelling. Applied Acoustics, Vol. 63, 2002, pp. 173-187.

[5] Gauvreau, B., Bérengier, M., Blanc-Benon, Ph., and Depollier, C. Traffic noise prediction with the parabolic equation method: Validation of a split-step PadÔ approach in complex environments. J. Acoust. Soc. Am. 112, 2680 (2002).

[6] Forssén J. Calculation of sound reduction by a screen in a turbulent atmosphere using the parabolic equation method. Acustica-Acta Acustica 1998, Vol. 84, pp. 599-606.

[7] Van Renterghem, T., Salomons, E. and Botteldooren, D. Parameter study of sound propagation between city canyons with coupled FDTD-PE model. Applied Acoustics, 67 (6), p. 487-510, 2006.

[8] Ögren, M. and Forssén J. Modelling of a city canyon problem in a turbulent atmosphere using an equivalent sources approach. Applied Acoustics, Vol. 65, 2004, pp. 629-642.

[9] Tatarskii VI The effects of the turbulent atmosphere on wave propagation. Keter Press, Jerusalem, 1971.

[10] Ishimaru, A. Wave propagation and scattering in random media. IEEE Press (and Oxford University Press, Oxford), New York, 1997.

[11] Ostashev VE. Acoustics in moving inhomogeneous media. E \& FN Spon (an imprint of Thomson Professional), London, 1997.

[12] Plovsing B. Proposal for Nordtest Method: Nord2000 - Prediction of Outdoor Sound Propagation. Nordtest Proposal AV 1106/07. 2007, Hørsholm, Denmark: Delta.

[13] van Maercke, D. and Defrance, J. Development of an Analytical Model for Outdoor Sound Propagation Within the Harmonoise Project Acustica-Acta Acustica, Vol. 93, 2007 , pp. 201-212.

[14] Forssén J. Influence of Atmospheric Turbulence on Sound Reduction by a Thin, Hard Screen: A Parameter Study Using the Sound Scattering Cross-Section. Proc. 8th Int. Symp. on Long-Range Sound Propagation, The Pennsylvania State University, USA, pp. 352-364 (1998).

[15] T. Van Renterghem, W. Wei, J. Forssén, M. Hornikx, M. Ögren, D. Botteldooren, E. Salomons. Improving the accuracy of engineering models at shielded building facades: experimental analysis of turbulence scattering. Proceedings Internoise 2013, Innsbruck, Austria.

[16] W. Wei, D. Botteldooren, T. Van Renterghem, M. Hornikx, J. Forssén, E. Salomons, M. Ögren. Urban background noise mapping: the general model. Submitted for publication in Acustica-Acta Acustica. 


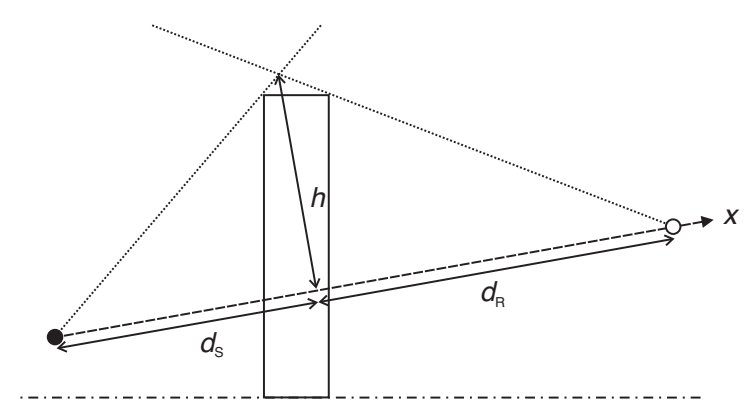

Figure 1. Geometric set-up for single noise barrier.

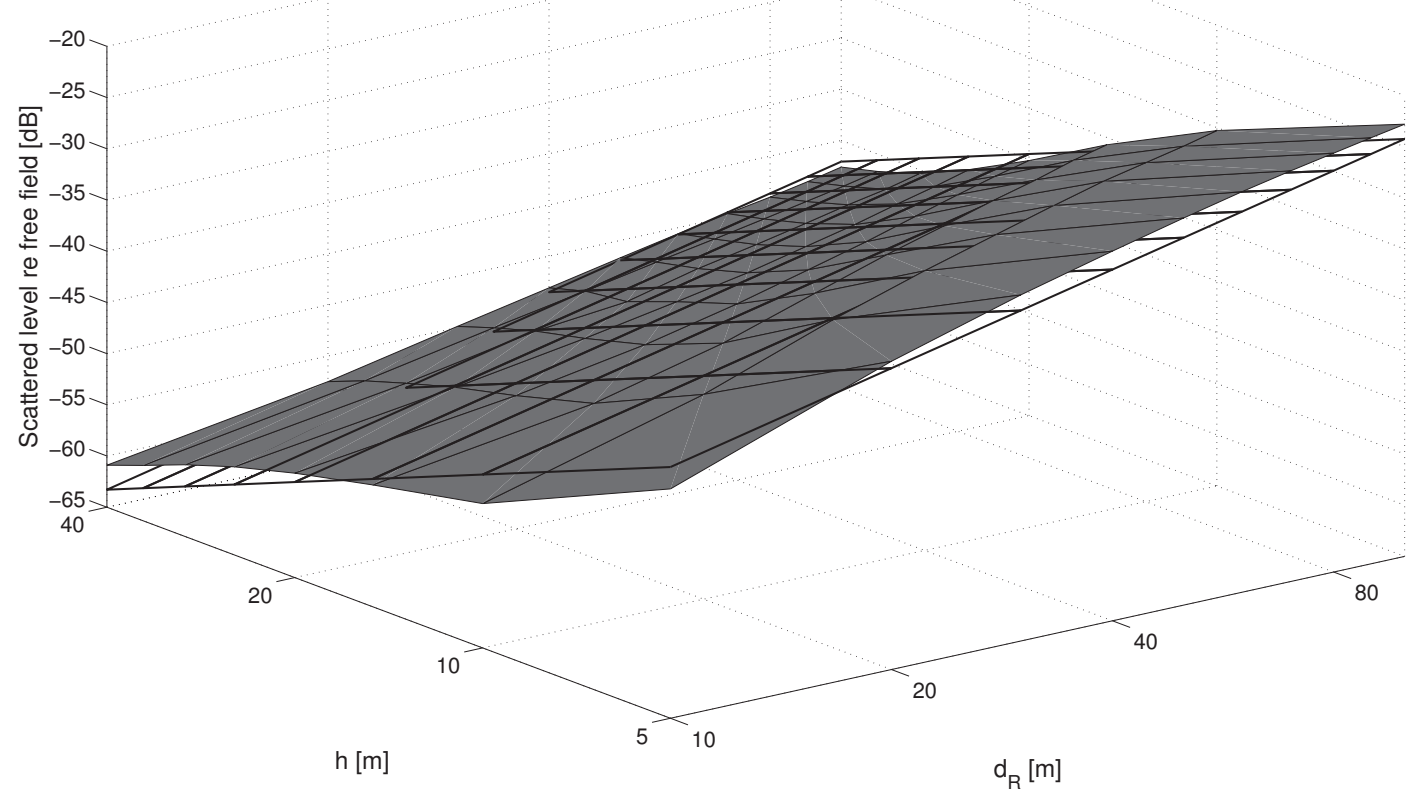

Figure 2. Comparson between originally calculated results (grayscale surface) and the best fit plane (black grid) for $f_{0}=1000 \mathrm{~Hz}, C_{v}^{2}=1 \mathrm{~m}^{4 / 3} / \mathrm{s}^{2}$ and $C_{T}^{2}=0$. 


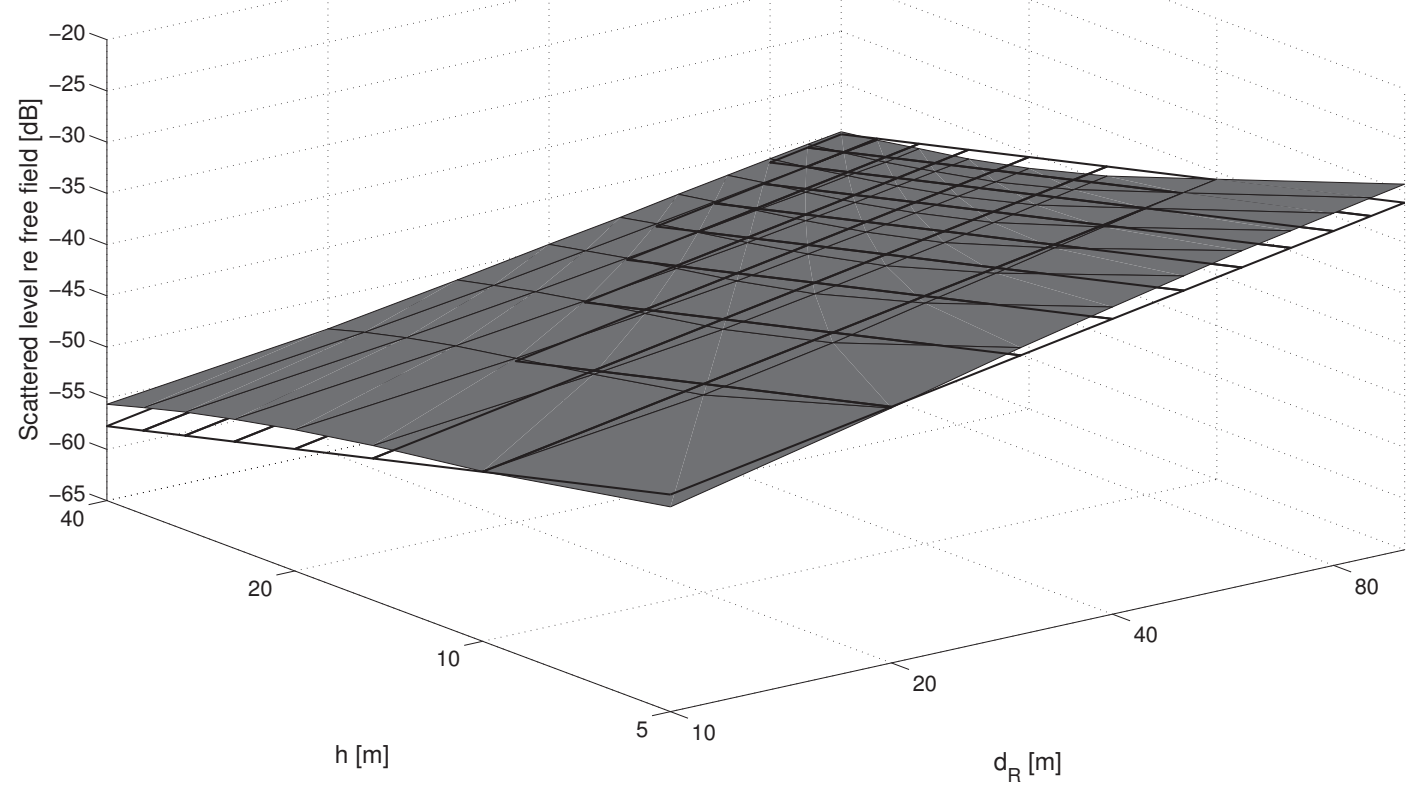

Figure 3. Comparson between originally calculated results (grayscale surface) and the best fit plane (black grid) for $f=1000 \mathrm{~Hz}, C_{v}^{2}=0$ and $C_{T}^{2}=1 \mathrm{~K}^{2} / \mathrm{m}^{2 / 3}$.

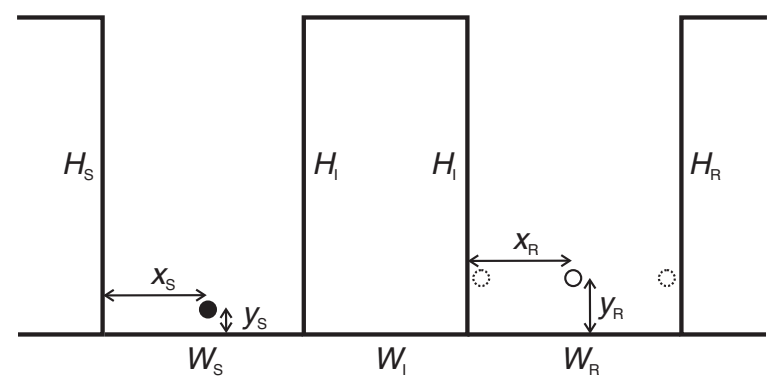

Figure 4. Geometric set-up for urban canyon situations. 
Table I. Linear fit coefficients for velocity and temperature turbulence.

\begin{tabular}{lll}
\hline \hline & $\begin{array}{l}\text { Velocity } \\
\text { turbulence }\end{array}$ & $\begin{array}{l}\text { Temperature } \\
\text { turbulence }\end{array}$ \\
\hline$b_{1}=$ & $-52.8+10 \log _{10} C_{v}^{2}$ & $-49.6+10 \log _{10} C_{T}^{2}$ \\
\hline$b_{2}=$ & 11.3 & 11.5 \\
\hline$b_{3}=$ & -17.1 & -13.1 \\
\hline \hline
\end{tabular}

Table II. Input data to parameter study of turbulence scattering for urban canyon situations. The geometric parameters are explained in Fig. 4. The last five parameters are the maximum reflection order, $m$, the façade's energy absorption coefficient, $\alpha$, the sound speed, $c_{0}$, the octave band centre frequencies, $f$, and the air attenuation, $\beta$.

\begin{tabular}{|c|c|c|c|c|c|c|c|c|c|c|}
\hline$H_{I}$ & $=$ & 5 & 10 & 20 & 40 & & & & & {$[\mathrm{~m}]$} \\
\hline$H_{S}, H_{R}$ & $=$ & 0 & $H_{I}$ & & & & & & & {$[\mathrm{~m}]$} \\
\hline$W_{I}$ & $=$ & .1 & 1 & 10 & 20 & 40 & 200 & & & {$[\mathrm{~m}]$} \\
\hline$W_{S}, W_{R}$ & $=$ & 5 & 10 & 20 & 40 & 80 & 160 & 320 & & {$[\mathrm{~m}]$} \\
\hline$x_{S}$ & $=$ & $.5 W_{S}$ & & & & & & & & {$[\mathrm{~m}]$} \\
\hline$x_{R}$ & $=$ & $.05 W_{R}$ & $.5 W_{R}$ & $.95 W_{R}$ & & & & & & {$[\mathrm{~m}]$} \\
\hline$y_{S}$ & $=$ & .5 & & & & & & & & {$[\mathrm{~m}]$} \\
\hline$y_{R}$ & $=$ & 1.5 & & & & & & & & {$[\mathrm{~m}]$} \\
\hline$m$ & $=$ & 15 & & & & & & & & {$[-]$} \\
\hline$\alpha$ & $=$ & .2 & & & & & & & & {$[-]$} \\
\hline$c_{0}$ & $=$ & 340 & & & & & & & & {$[\mathrm{~m} / \mathrm{s}]$} \\
\hline$f$ & $=$ & 31.5 & 63 & 125 & 250 & 500 & $1 \mathrm{k}$ & $2 \mathrm{k}$ & $4 \mathrm{k}$ & {$[\mathrm{Hz}]$} \\
\hline$\beta$ & $=$ & .023 & .090 & .34 & 1.1 & 2.8 & 5.0 & 9.0 & 23 & {$[\mathrm{~dB} / \mathrm{km}]$} \\
\hline
\end{tabular}




\section{A. Tabulated coefficients used for pa- rameter study}

Table III. Values of coefficients to define the planes of scattered levels for a unit strength of velocity turbulence, i.e. $C_{v}^{2}=1 \mathrm{~m}^{4 / 3} / \mathrm{s}^{2}$ and $C_{T}^{2}=0$, at $f=1000 \mathrm{~Hz}$, for varying values of the source-screen distance, $d_{S}$. The scattered level relative to free field is $L_{p \text {,scat }}=a_{1}+a_{2} \log _{10}\left(d_{R} / d_{S}\right)+$ $a_{3} \log _{10}\left(h / d_{S}\right) \mathrm{dB}$, where $d_{R}$ is the screen-receiver distance and $h$ is the screen height. For intermediate values of $d_{S}$, interpolation is used.

\begin{tabular}{llll}
\hline$d_{S}$ & $a_{1}$ & $a_{2}$ & $a_{3}$ \\
{$[\mathrm{~m}]$} & {$[\mathrm{dB}]$} & {$[\mathrm{dB}]$} & {$[\mathrm{dB}]$} \\
\hline \hline 10.0 & -61.6 & 17.9 & -19.5 \\
11.8 & -60.2 & 17.6 & -20.5 \\
13.9 & -59.0 & 17.3 & -21.4 \\
16.3 & -57.9 & 17.1 & -22.4 \\
19.2 & -56.9 & 16.8 & -23.2 \\
22.6 & -56.0 & 16.6 & -23.9 \\
26.6 & -55.3 & 16.4 & -24.5 \\
31.3 & -54.5 & 16.3 & -24.9 \\
36.8 & -53.9 & 16.2 & -25.2 \\
43.4 & -53.2 & 16.2 & -25.3 \\
51.0 & -52.5 & 16.2 & -25.3 \\
60.1 & -51.8 & 16.3 & -25.2 \\
70.7 & -51.0 & 16.4 & -25.0 \\
83.2 & -50.1 & 16.6 & -24.7 \\
98.0 & -49.1 & 16.7 & -24.3 \\
115 & -48.0 & 16.9 & -23.8 \\
136 & -46.8 & 17.1 & -23.3 \\
160 & -45.5 & 17.3 & -22.7 \\
188 & -44.2 & 17.4 & -22.2 \\
221 & -42.9 & 17.6 & -21.7 \\
261 & -41.5 & 17.7 & -21.2 \\
307 & -40.2 & 17.8 & -20.7 \\
361 & -38.9 & 17.9 & -20.3 \\
425 & -37.6 & 17.9 & -19.9 \\
500 & -36.3 & 18.0 & -19.5 \\
\hline \hline & & &
\end{tabular}

Table IV. Same as in Table III except for a unit strength of temperature turbulence, i.e. $C_{T}^{2}=1 \mathrm{~K}^{2} / \mathrm{m}^{2 / 3}$ and $C_{v}^{2}=0$.

\begin{tabular}{llll}
\hline$d_{S}$ & $a_{1}$ & $a_{2}$ & $a_{3}$ \\
{$[\mathrm{~m}]$} & {$[\mathrm{dB}]$} & {$[\mathrm{dB}]$} & {$[\mathrm{dB}]$} \\
\hline \hline 10.0 & -58.3 & 14.9 & -11.6 \\
11.8 & -57.1 & 14.5 & -12.0 \\
13.9 & -56.0 & 14.2 & -12.5 \\
16.3 & -54.9 & 13.9 & -13.0 \\
19.2 & -53.8 & 13.6 & -13.5 \\
22.6 & -52.9 & 13.3 & -14.0 \\
26.6 & -52.0 & 13.1 & -14.5 \\
31.3 & -51.2 & 12.9 & -14.9 \\
36.8 & -50.5 & 12.8 & -15.3 \\
43.4 & -49.7 & 12.6 & -15.6 \\
51.0 & -49.1 & 12.6 & -15.8 \\
60.1 & -48.4 & 12.5 & -16.0 \\
70.7 & -47.7 & 12.5 & -16.1 \\
83.2 & -47.0 & 12.5 & -16.1 \\
98.0 & -46.2 & 12.5 & -16.1 \\
115 & -45.4 & 12.6 & -16.0 \\
136 & -44.6 & 12.6 & -15.8 \\
160 & -43.6 & 12.7 & -15.6 \\
188 & -42.7 & 12.8 & -15.4 \\
221 & -41.7 & 12.8 & -15.2 \\
261 & -40.6 & 12.9 & -14.9 \\
307 & -39.6 & 12.9 & -14.7 \\
361 & -38.5 & 13.0 & -14.4 \\
425 & -37.4 & 13.0 & -14.2 \\
500 & -36.4 & 13.0 & -13.9 \\
\hline \hline & & &
\end{tabular}

